\title{
Low Rank Cohomology of the Classifying Spaces of Gauge Groups over 3-manifolds
}

By

\author{
Shizuo KAJI*
}

\begin{abstract}
The purpose of this paper is to calculate the cohomology of the function space $\operatorname{Map}(M, B G)$ for degree less than or equal to 3 , where $G$ is a simply connected compact Lie group and $M$ is a closed orientable 3-manifold. The calculation enables us to obtain a simple proof and an improvement of the result [4, Theorem 1.2].
\end{abstract}

\section{$\S 1$. Introduction}

Let $G$ be a simply connected compact Lie group and $M$ a closed orientable 3 -manifold. Since $B G$ is 3-connected, any principal $G$-bundles over $M$ are trivial. Then we call the gauge group of the trivial $G$-bundle over $M$ the gauge group over $M$ and denote it by $\mathcal{G}$. It is well-known that

$$
B \mathcal{G} \simeq \operatorname{Map}(M, B G)
$$

([2]). The cohomology of $B \mathcal{G}$ in low dimensions is considered in [4] by making use of the Eilenberg-Moore spectral sequence.

Theorem 1.1 [4, Theorem 1.2]. Suppose that $\operatorname{Tor}_{\mathbb{Z}}(\mathbb{Z} / 2, R)=0$. Let $G$ be a simply-connected compact Lie group such that the integral cohomology of $B G$ is torsion free and let $M$ be a closed orientable 3-manifold. We denote $H^{i}(\operatorname{Map}(M, B G) ; R)$ by $H^{i}$. Then there exists a short exact sequence

$$
0 \rightarrow H_{1}(M ; R)^{\oplus r} \oplus R^{\oplus s} \oplus H^{1} \otimes H^{2} \stackrel{\alpha}{\rightarrow} H^{3} \rightarrow(R / 2 R)^{\oplus r} \rightarrow 0,
$$

where $\left.\alpha\right|_{H^{1} \otimes H^{2}}$ is the cup product, $r=\operatorname{rank} H^{4}(B G)$ and $s=\operatorname{rank} H^{6}(B G)$. Moreover $H^{1}$ is a free $R$-module for any $R$, and $H^{2}$ is also free if $R$ is a PID.

Communicated by K. Saito. Received December 17, 2004. Revised March 25, 2005.

2000 Mathematics Subject Classification(s): 55R40, 54C35

*Department of Mathematics, Kyoto University, Kyoto 606-8502, Japan. 
The purpose of this paper is to refine Theorem 1.1 and to give a simple proof. We determine the integral cohomology of $H^{i}(\operatorname{Map}(M, B G))(i \leq 3)$ without the assumption that $H^{*}(B G)$ is torsion free. It is known that $G$ is the direct product of simply connected compact simple Lie groups $([6])$. Then we reduce Theorem 1.1 to the case that $G$ is a simply connected compact simple Lie group and obtain

Theorem 1.2. $\quad$ Let $G$ be a simply connected compact simple Lie group and $M$ a closed orientable 3-manifold. We denote $H^{i}(\operatorname{Map}(M, B G))$ by $H^{i}$. Then we have

$$
H^{i} \cong \begin{cases}\mathbb{Z} & i=0 \\ H^{1}\left(\Omega^{2} G\right) & i=1 \\ H_{2}(M) & i=2 \\ H^{1}\left(\Omega^{2} G\right) \otimes H_{2}(M) \oplus H_{1}(M) \oplus H^{3}\left(\Omega^{2} G\right) & i=3 .\end{cases}
$$

Moreover, the cup product $H^{1} \otimes H^{2} \rightarrow H^{3}$ maps $H^{1} \otimes H^{2}$ isomorphically onto the direct summand $H^{1}\left(\Omega^{2} G\right) \otimes H_{2}(M) \subset H^{3}$.

Remark. Let $G$ be a simply connected compact simple Lie group. We here describe the integral cohomology $H^{*}\left(\Omega^{2} G\right)$ for $* \leq 3$.

Table $1 . H^{*}\left(\Omega^{2} G\right)$

\begin{tabular}{|c|ccc|}
\hline & \multicolumn{3}{|c|}{ type of $G$} \\
$H^{i}\left(\Omega^{2} G\right)$ & $A_{l}(l \geq 2)$ & $C_{l}(l \geq 1)$ & otherwise \\
\hline$i=1$ & $\mathbb{Z}$ & $\mathbb{Z}$ & $\mathbb{Z}$ \\
$i=2$ & 0 & 0 & 0 \\
$i=3$ & $\mathbb{Z}$ & $\mathbb{Z} / 2$ & 0 \\
\hline
\end{tabular}

Remark. Since the inclusion of the 1-skeleton $\bigvee^{g-1} S^{1} \rightarrow \overbrace{\mathbb{R} P^{2} \# \cdots \# \mathbb{R} P^{2}}^{g}$ induces an isomorphism on $\bmod p$ cohomology for each odd prime $p$, Theorem 1.1 in [4] is easily shown without the assumption that $H^{*}(G)$ is $p$-torsion free by [5, Proposition 4.2] and [3, Ch. VI, Proposition 7.1]. 


\section{$\S 2 . \quad$ Approximation of $G$ by Infinite Loop Spaces}

Let $G$ be a simply connected compact Lie group. In this section we approximate $G$ by an infinite loop space in low dimensions.

It is known that $G$ is the direct product of simply connected compact simple Lie groups. Since simply connected compact simple Lie groups are classified by their Lie algebras as $A_{l}, B_{l}, C_{l}, D_{l}(l \geq 1), E_{l}(l=6,7,8), F_{4}, G_{2}$, we give an approximation to each type.

Proposition 2.1. Let $G$ be a simply connected compact simple Lie group. Then there exist an infinite loop space $\mathbf{B}$ and a 7 -equivalence $t: B G \rightarrow$ B.

Proof. First we note that there are the following correspondence in low $\operatorname{ranks}([6])$ :

$$
A_{1}=B_{1}=C_{1}, B_{2}=C_{2}, D_{2}=A_{1} \times A_{1}, D_{3}=A_{3} .
$$

Therefore we have only to consider the types $A_{l}(l \geq 2), B_{l}(l \geq 3), C_{l}(l \geq$ $1), D_{l}(l \geq 4), E_{l}(l=6,7,8), F_{4}$ and $G_{2}$. In the case where $G$ is of type $A_{l}(l \geq 2)$, there exists a 7 -equivalence $B G \rightarrow B S U$ induced by the inclusion $G \rightarrow S U$. Similarly in the case where $G$ is of type $C_{l}(l \geq 1)$, there exists a 7 -equivalence $B G \rightarrow B S p$. For $G$ otherwise, we have $\pi_{i}(G)=0(i=1,2,4,5)([6])$. Then a representative of a generator of $H^{4}(B G ; \mathbb{Z}) \cong \mathbb{Z}$ is a 7 -equivalence $B G \rightarrow$ $K(\mathbb{Z}, 4)$.

Corollary 2.1. Let $M$ be a 3-dimensional complex. Then we have $H^{i}(\operatorname{Map}(M, \mathbf{B})) \cong H^{i}(\operatorname{Map}(M, B G))(i \leq 3)$, where $\mathbf{B}$ is as in Proposition 2.1.

Proof. Let $\operatorname{Map}_{*}(X, Y)$ denote the space of basepoint preserving maps from $X$ to $Y$, where $X, Y$ are based spaces.

We consider the following commutative diagram

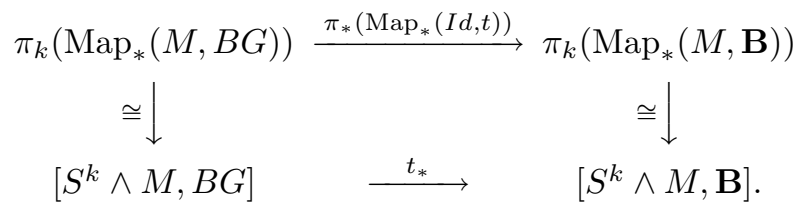

Since the second row is an isomorphism for $k \leq 3$ and a surjection for $k=4$ by J.H.C. Whitehead theorem, we see that $\operatorname{Map}_{*}(I d, t): \operatorname{Map}_{*}(M, B G) \rightarrow$ 
$\operatorname{Map}_{*}(M, \mathbf{B})$ is a 4-equivalence. Consider the following commutative diagram of evaluation fibrations

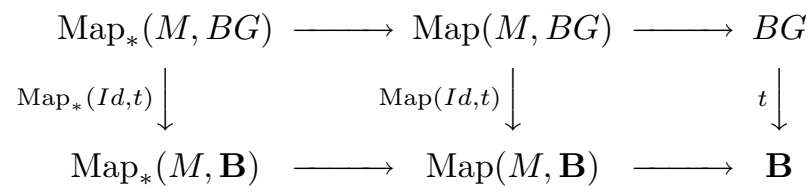

Since $\operatorname{Map}_{*}(I d, t)$ is a 4-equivalence and $t$ is a 7 -equivalence, it follows that $\operatorname{Map}(I d, t): \operatorname{Map}(M, B G) \rightarrow \operatorname{Map}(M, \mathbf{B})$ is a 4-equivalence.

\section{$\S 3 . \quad$ Proof of Theorem 1.2}

Let $G$ be a simply connected compact simple Lie group. By Proposition 2.1 there exists an infinite loop space $\mathbf{B}$ and a 7 -equivalence $t: B G \rightarrow \mathbf{B}$. Since $\mathbf{B}$ is a homotopy group, we have a homotopy equivalence

$$
\begin{aligned}
\operatorname{Map}(M, \mathbf{B}) & \simeq \operatorname{Map}_{*}(M, \mathbf{B}) \times \mathbf{B} \\
f & \mapsto\left(f \cdot f(*)^{-1}, f(*)\right),
\end{aligned}
$$

where $*$ denotes the basepoint of $M$. Since the infinite loop space $\mathbf{B}$ is 3connected, the inclusion map $\operatorname{Map}_{*}(M, B) \rightarrow \operatorname{Map}(M, B)$ induces the isomorphism on homology for degree less than or equal to 3 . Then we compute $H^{*}\left(\operatorname{Map}_{*}(M, \mathbf{B})\right)$ to determine $H^{*}(\operatorname{Map}(M, \mathbf{B}))$.

Proposition 3.1. We have

$$
H^{k}\left(\operatorname{Map}_{*}(M, \mathbf{B})\right) \cong \bigoplus_{i+j=k} H^{i}\left(\operatorname{Map}_{*}\left(M^{2}, \mathbf{B}\right)\right) \otimes H^{j}\left(\Omega^{3} \mathbf{B}\right)
$$

for $k \leq 3$, where $M^{2}$ is the 2-skeleton of $M$.

Proof. Since a closed orientable 3-manifold is parallelizable, the top cell of $M$ is split off stably ([1]). Actually by Freudenthal suspension theorem the top cell of $M$ is split off after double suspension. Then we have

$$
\begin{aligned}
\operatorname{Map}_{*}(M, \mathbf{B}) & \left.\simeq \operatorname{Map}_{*}\left(M, \Omega^{2} B^{2} \mathbf{B}\right)\right) \\
& \simeq \operatorname{Map}_{*}\left(\Sigma^{2} M, B^{2} \mathbf{B}\right) \\
& \simeq \operatorname{Map}_{*}\left(\Sigma^{2} M^{2} \vee S^{5}, B^{2} \mathbf{B}\right) \\
& \simeq \operatorname{Map}_{*}\left(M^{2} \vee S^{3}, \mathbf{B}\right) \\
& \simeq \operatorname{Map}_{*}\left(M^{2}, \mathbf{B}\right) \times \Omega^{3} \mathbf{B} .
\end{aligned}
$$


Since $H^{k}\left(\Omega^{3} \mathbf{B}\right)(k<3)$ is either 0 or $\mathbb{Z}$, the proof is completed by Künneth Theorem.

To compute $H^{i}\left(\operatorname{Map}_{*}\left(M^{2}, \mathbf{B}\right)\right)(i \leq 3)$ we need the following technical lemma. Let $X, Y, Z$ be based spaces and $f: X \rightarrow Y$ be a based map. We denote by $f^{\#}$ the induced map $\operatorname{Map}_{*}(f, I d): \operatorname{Map}_{*}(Y, Z) \rightarrow \operatorname{Map}_{*}(X, Z)$.

Lemma 3.1. $\quad$ Let $X$ be a based space such that there is a $(p+q+1)$ equivalence $g: X \rightarrow K(\mathbb{Z}, p+q)$ and let $f: \bigvee^{l} S^{p} \rightarrow \bigvee^{m} S^{p}$ be a based map. Suppose $\left(f^{\#}\right)^{*}: H^{q}\left(\Pi^{l} \Omega^{p} X\right) \rightarrow H^{q}\left(\Pi^{m} \Omega^{p} X\right)$ is represented by a matrix $A$ for a certain basis. Then $f_{*}: H_{p}\left(\bigvee^{l} S^{p}\right) \rightarrow H_{p}\left(\bigvee^{m} S^{p}\right)$ is also represented by $A$ for a suitable basis.

Proof. Since $g_{*}:\left[S^{p+q}, X\right] \rightarrow\left[S^{p+q}, K(\mathbb{Z}, p+q)\right]$ is an isomorphism, we have the following commutative diagram

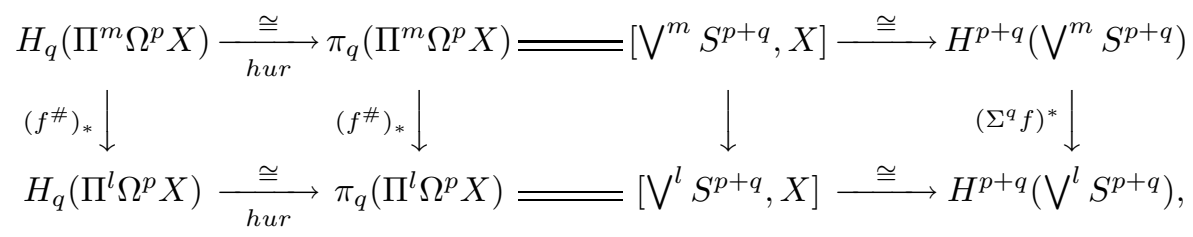

where hur is the Hurewicz homomorphism. Since $\Omega^{p} X \rightarrow K(\mathbb{Z}, q)$ is a $(q+1)$ equivalence, the proof is completed by taking the dual.

Proposition 3.2. $H^{i}\left(\operatorname{Map}_{*}\left(M^{2}, \mathbf{B}\right)\right) \cong \begin{cases}0 & i=1 \\ H_{2}(M) & i=2 \\ H_{1}(M) & i=3\end{cases}$

Proof. We have the following cofibration sequence $\bigvee^{l} S^{1} \stackrel{f}{\rightarrow} \bigvee^{m} S^{1} \stackrel{i}{\rightarrow}$ $M^{2} \rightarrow \bigvee^{l} S^{2}$, where $f$ is the attaching map of 2-cells of $M$ and $i$ is the inclusion. Then we have the fibration

$$
\Pi^{l} \Omega^{2} \mathbf{B} \rightarrow \operatorname{Map}_{*}\left(M^{2}, \mathbf{B}\right) \stackrel{i^{\#}}{\longrightarrow} \Pi^{m} \Omega \mathbf{B} .
$$

We consider the Leray-Serre spectral sequence $\left(E_{r}, d_{r}\right)$ of the fibration above. Since $E_{2}^{p, q} \cong H^{p}\left(\Pi^{m} \Omega \mathbf{B}\right) \otimes H^{q}\left(\Pi^{l} \Omega^{2} \mathbf{B}\right), \mathbf{B}$ is 3 -connected and $H^{4}(\mathbf{B}) \cong \mathbb{Z}$, the non-trivial differential $d_{r}: E_{r}^{p, q} \rightarrow E_{r}^{p+r, q-r+1}(p+q \leq 4)$ occurs only when $r=3$ and $(p, q)=(0,2)$. Then we obtain $H^{1}\left(\operatorname{Map}_{*}\left(M^{2}, \mathbf{B}\right)\right)=0$. Next we determine $d_{3}: E_{3}^{0,2} \rightarrow E_{3}^{3,0}$ to compute $H^{i}\left(\operatorname{Map}_{*}\left(M^{2}, \mathbf{B}\right)\right)(i=2,3)$. We 
consider the commutative diagram

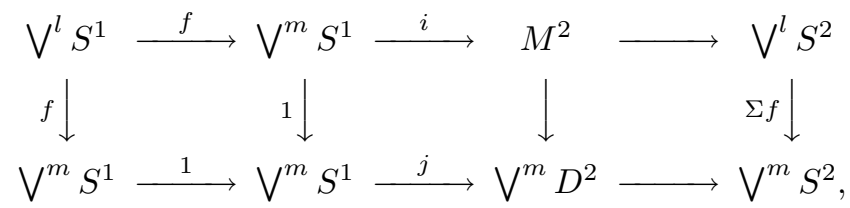

where $j$ is the inclusion. Applying $\operatorname{Map}_{*}(, \mathbf{B})$ to above, we have the following commutative diagram

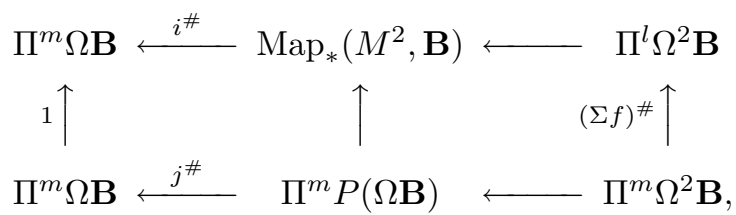

where the second row is the product of the path space fibrations of $\Omega \mathbf{B}$. Comparing the Leray-Serre spectral sequence of fibrations above, we obtain $d_{3}=\tau(\Sigma f)^{\# *}: E_{3}^{0,2} \rightarrow E_{3}^{3,0}$, where $\tau: H^{2}\left(\Pi^{m} \Omega^{2} \mathbf{B}\right) \stackrel{\cong}{\leftrightarrows} H^{3}\left(\Pi^{m} \Omega \mathbf{B}\right)$ is the transgression.

Let $A$ be a matrix which represents $\left((\Sigma f)^{\#}\right)^{*}: H^{2}\left(\Pi^{l} \Omega^{2} \mathbf{B}\right) \rightarrow H^{2}\left(\Pi^{m} \Omega^{2} \mathbf{B}\right)$. By Lemma 3.1, $(\Sigma f)_{*}: H_{2}\left(\bigvee^{l} S^{2}\right) \rightarrow H_{2}\left(\bigvee^{m} S^{2}\right)$ is represented by $A$ and so is $f_{*}: H_{1}\left(\bigvee^{l} S^{1}\right) \rightarrow H_{1}\left(\bigvee^{m} S^{1}\right)$. Then we have the exact sequence

$$
0 \rightarrow H_{2}\left(M^{2}\right) \rightarrow H_{1}\left(\bigvee^{l} S^{1}\right) \stackrel{A}{\rightarrow} H_{1}\left(\bigvee^{m} S^{1}\right) \rightarrow H_{1}\left(M^{2}\right) \rightarrow 0
$$

Since $H_{i}\left(M^{2}\right) \cong H_{i}(M)(i \leq 2)$, we have

$$
\begin{aligned}
& H^{2}\left(\operatorname{Map}_{*}\left(M^{2}, \mathbf{B}\right)\right) \cong \operatorname{Ker}\left\{d_{3}: E_{3}^{0,2} \rightarrow E_{3}^{3,0}\right\} \cong \operatorname{Ker} A \cong H_{2}(M), \\
& H^{3}\left(\operatorname{Map}_{*}\left(M^{2}, \mathbf{B}\right)\right) \cong \operatorname{Coker}\left\{d_{3}: E_{3}^{0,2} \rightarrow E_{3}^{3,0}\right\} \cong \operatorname{Coker} A \cong H_{1}(M) .
\end{aligned}
$$

Proof of Theorem 1.2. By Corollary 2.1, Proposition 3.1 and Proposition 3.2 , Theorem 1.2 is proved.

\section{Acknowledgment}

I would like to thank Akira Kono and Daisuke Kishimoto for their helpful conversation and useful comments. 


\section{References}

[1] Atiyah, M., Thom complexes, Proc. London Math. Soc. (3), 11 (1961), 291-310.

[2] Atiyah, M. and Bott, R., The Yang-Mills equations over Riemann surfaces, Phils. Trans. Ray. Soc. London Ser A., 308 (1982), 523-615.

[3] Bousfield, A. K. and Kan, D. M., Homotopy limits, completions and localizations, Lecture Notes in Math., 304, Springer-Verlag, Berlin and New York (1972).

[4] Kuribayashi, K., Eilenberg-Moore spectral sequence calculation of function space cohomology, Manuscripta Math., 114 (2004), 305-325.

[5] May, J., Fibrewise localization and completion, Trans. Amer. Math. Soc., 258 (1980), 127-146.

[6] Mimura, M. and Toda, H., Topology of Lie groups. I, II, Translated from the 1978 Japanese edition by the authors, Transl. Math. Monogr., 91, Amer. Math. Soc., Providence, RI (1991). 